\title{
DETERMINAÇÃO DE DADOS DE EQUILÍBRIO LÍQUIDO-VAPOR A ALTA PRESSÃO PARA O SISTEMA DIÓXIDO DE CARBONO + ACETATO DE ETILA + ÁCIDO OLEICO
}

\author{
A. F. ZANETTE, L. FERREIRA-PINTO, J. C. LIMA, L. CARDOZO FILHO \\ Universidade Estadual de Maringá, Departamento de Engenharia Química \\ E-mail para contato: andreiazanette@yahoo.com.br
}

\begin{abstract}
RESUMO - O objetivo geral deste trabalho é a obtenção de epóxidos a partir do ácido oleico utilizando dióxido de carbono em condições supercríticas como solvente. Para atingir o objetivo, foram obtidos os dados de transição de fase para o sistema ternário $\left\{\mathrm{CO}_{2}(1)+\right.$ acetato de etila(2) + ácido oleico(3) $\}$ em diferentes frações molares de ácido acetato de etila:oleico. Uma célula com janelas frontais e laterais e de volume variável foi utilizada para obter os dados experimentais. $\mathrm{O}$ intervalo de temperatura foi 35 a $65^{\circ} \mathrm{C}$ e pressões de até $22 \mathrm{MPa}$ foram atingidas. Foram observadas transições de fase líquidovapor do tipo ponto de bolha e, em concentrações de dióxido de carbono mais elevadas, foram observadas transições tipo ponto de orvalho.
\end{abstract}

\section{INTRODUÇÃO}

A epoxidação é uma reação muito útil em síntese orgânica. Devido à elevada reatividade do grupo epóxido, e pode ser utilizada como um intermediário ativo para a síntese de muitos produtos de alto valor comercial tais como álcoois, glicóis, alcaloaminas, compostos de carbonil, compostos olefínicos e polímeros como poliésteres, surfactantes de alta tecnologia, poliuretanos e resinas epoxi (Dinda et al.,, 2011, Rios et al.,, 2005).

Classicamente, a síntese de um epóxido é realizada por oxidação de um ácido orgânico e peróxido de hidrogênio (portador de oxigênio) que gera um perácido. As reações pioneiras nesta área posteriormente levaram o nome de seus inventores, que são a reação de epoxidação de Prilezhaev e a reação de Baeyer-Villiger (Kotlewska et al.,, 2011). Recentemente, a epoxidação enzimática tem sido desenvolvida com êxito, mostrando-se superior à síntese química (Petrovic et al.,, 2002, Sun et al., 2011). As condições de reação são suaves, ocorre a formação de hidroperóxidos estáveis diretamente a partir de ácidos graxos, as enzimas apresentam alta, regio e estereosselectividade, suprindo significativamente reações secundárias, obtendo conversões elevadas (Correa et al.,, 2012; Lu et al., 2010).

Diversos trabalhos encontrados na literatura têm estudado a epoxidação de diferentes ácidos graxos e óleos de plantas utilizando catalisadores químicos (Saithai et al.,, 2013; Dinda et al.,, 2011; Mushtaq et al.,, 2013; Roumanet et al.,, 2013) e enzimáticos (Correa et al.,, 2012; Sun et al.,, 2011; Silva et al.,, 2011; Kotlewska et al.,, 2011; Saithai et al.,, 2013). 


\section{9 a 22 de outubro de 2014 \\ Florianópolis/SC}

O dióxido de carbono em condições supercríticas tem sido utilizado como solvente na extração de materiais bioativos e, mais recentemente, na síntese de novos produtos. As baixas temperatura e pressão críticas, sua não toxicidade e por não ser inflamável oferece grande vantagem em relação aos outros solventes (Akin e Temelli, 2012; Al- Darmaki et al., 2011). O conhecimento do efeito da temperatura, pressão e composição do comportamento de fase de um sistema reacional é fundamental para determinar as condições mais favoráveis para o processo de síntese (Laudani et al., 2009). Para o sistema em questão, a obtenção de epóxidos por meio da epoxidação enzimática em $\mathrm{CO}_{2}$ supercrítico ainda não foi investigada.

Para aprofundar a compreensão desta nova técnica de síntese de epóxidos, é importante obter os dados de equilíbrio de fases para o sistema ternário selecionado. Assim, este trabalho relata dados de equilíbrio para o sistema dióxido de carbono(1) + acetato de etila(2) + ácido oleico(3).

\section{EXPERIMENTAL}

\subsection{Materiais}

Acetato de etila padrão analítico foi obtido da Sigma-Aldrich. Ácido oleico padrão analítico foi obtido da Vetec. Dióxido de carbono foi obtido da White Martins S.A. Todas as substâncias foram utilizadas sem nenhum tratamento prévio.

\subsection{Aparato de Equilíbrio de Fase e Procedimento Experimental}

As medidas experimentais de equilíbrio de fases a altas pressões foram baseadas no método estático sintético e o aparato e metodologia experimental utilizado são similares ao proposto por Pinto et al., (2013), Santos et al., (2012), Mazzer et al., (2012) e Giufrida et al., (2011). O aparato experimental é composto de uma bomba volumétrica de alta-pressão do tipo seringa (ISCO 260D) e uma célula volumétrica de alta pressão com capacidade de $27 \mathrm{~cm}^{3}$, de volume variável e com janelas lateral e frontal de safira. As condições operacionais foram controladas por meio de um controlador de pressão absoluto com precisão de 0,03 MPa (Smar LD 301) e controlador de temperatura ligado a um termopar (tipo T, precisão de $\pm 1,0 \mathrm{~K}$ ), que esteve em contato com o sistema no interior da célula. Internamente, a célula de equilíbrio é composta por um pistão móvel, que permite a variação da pressão, controlada pela bomba do tipo seringa.

Ao início de cada experimento, a célula foi purgada com $\mathrm{CO}_{2}$ para remoção do ar residual. A alimentação da célula (acetato de etila + ácido oleico) foi realizada por meio da injeção com uma seringa, pela abertura da conexão da linha de alimentação, à temperatura ambiente. Com o reator fechado inicia-se a alimentação do reator com o solvente. A quantidade exata de $\mathrm{CO}_{2}$ a ser alimentado é calculada a partir de sua densidade e a pressão do sistema é calculada pela equação de PengRobinson. A temperatura é então fixada e o sistema pressurizado até se manter monofásico. Em seguida, para obtenção dos dados de equilíbrio do tipo LV diminuiu-se a pressão do sistema gradativamente até o surgimento de uma nova fase. As medidas de transição foram realizadas para as temperaturas de $35,45,55$ e $65^{\circ} \mathrm{C}$, nas frações molares de ácido oleico:acetato de etila de 1:3, 1:9 e 1:200. Os dados foram obtidos com base em três repetições de cada ponto experimental. 


\section{RESULTADOS EXPERIMENTAIS}

Foram obtidos dados experimentais de equilíbrio de fases para o seguinte sistema ternário: $\mathrm{CO}_{2}$ + acetato de etila + ácido oleico a $35^{\circ} \mathrm{C}, 45^{\circ} \mathrm{C}, 55^{\circ} \mathrm{C}$ e $65^{\circ} \mathrm{C}$, nas frações molares de ácido oleico:acetato de etila de 1:3, 1:9 e 1:200 (Tabela 1). Esta última fração é bastante elevada e, pensando em fins industriais, pouco praticáveis, já que ocorre a formação de uma pequena quantidade de epóxido para um excesso muito grande de solvente. Os dados experimentais são apresentados em termos de fração molar de $\mathrm{CO}_{2}$ (x1), fração molar de acetato de etila (x2), pressão de transição de fase (P) e o desvio padrão das replicatas $\mathrm{u}(\mathrm{p})$.

$\mathrm{Na}$ fração molar de 1:3 $\left\{\mathrm{CO}_{2}(1)+(0,75\right.$ acetato de etila(2) $+0,25$ ácido oleico(3) $\}$ foram verificadas transições de fases líquido-vapor (ELV) do tipo ponto de bolha (PB) e, em frações de $\mathrm{CO}_{2}$ acima de 0,9 ocorreu a formação tipo ponto de orvalho (PO). Na fração molar de $1: 9\left\{\mathrm{CO}_{2}(1)+(0,90\right.$ acetato de etila(2) + 0,10 ácido oleico(3) \} foram verificadas transições de fases líquido-vapor (ELV) do tipo PB para todas as frações. Na fração molar de 1:200 $\left\{\mathrm{CO}_{2}(1)+(0,995\right.$ acetato de etila $(2)+$ 0,005 ácido oleico(3)\} foram verificadas transições de fases líquido-vapor (ELV) do tipo PB e, em frações de $\mathrm{CO}_{2}$ acima de 0,85 ocorreu a formação tipo PO.

Tabela 1 - Dados de equilíbrio de fase para o sistema ternário

\begin{tabular}{|c|c|c|c|c|c|c|c|c|c|}
\hline $\mathrm{x}_{1}$ & $\mathrm{x}_{2}$ & $p / \mathrm{MPa}$ & $u(p) / \mathrm{MPa}$ & Tipo $^{b}$ & $\mathrm{x}_{1}$ & $\mathrm{x}_{2}$ & $p / \mathrm{MPa}$ & $u(p) / \mathrm{MPa}$ & Tipo $^{b}$ \\
\hline \multicolumn{10}{|c|}{ Fração molar AO:acetato etila de 1:3 } \\
\hline \multicolumn{5}{|c|}{$T=35^{\circ} \mathrm{C}$} & \multicolumn{5}{|c|}{$T=45^{\circ} \mathrm{C}$} \\
\hline 0,50 & 0,37 & 3,44 & 0,02 & ELV (PB) & 0,50 & 0,37 & 3,24 & 0,01 & VLE (PB) \\
\hline 0,60 & 0,30 & 4,00 & 0,03 & ELV (PB) & 0,60 & 0,30 & 4,06 & 0,01 & VLE (PB) \\
\hline 0,70 & 0,22 & 5,06 & 0,03 & ELV (PB) & 0,70 & 0,22 & 4,98 & 0,02 & VLE (PB) \\
\hline 0,80 & 0,15 & 5,20 & 0,02 & ELV (PB) & 0,80 & 0,15 & 6,24 & 0,03 & VLE (PB) \\
\hline 0,90 & 0,075 & 12,62 & 0,01 & ELV (PO) & 0,90 & 0,075 & 13,55 & 0,02 & VLE (PO) \\
\hline 0,95 & 0,038 & 13,16 & 0,01 & ELV (PO) & 0,95 & 0,038 & 15,50 & 0,01 & VLE (PO) \\
\hline \multicolumn{5}{|c|}{$T=55^{\circ} \mathrm{C}$} & \multicolumn{4}{|c|}{$T=65^{\circ} \mathrm{C}$} & \\
\hline 0,50 & 0,37 & 3,81 & 0,03 & ELV (PB) & 0,50 & 0,37 & 4,50 & 0,02 & $\mathrm{ELV}(\mathrm{PB})$ \\
\hline 0,60 & 0,30 & 5,09 & 0,03 & ELV (PB) & 0,60 & 0,30 & 5,85 & 0,03 & $\operatorname{ELV}(\mathrm{PB})$ \\
\hline 0,70 & 0,22 & 6,17 & 0,02 & ELV (PB) & 0,70 & 0,22 & 7,45 & 0,02 & $\operatorname{ELV}(\mathrm{PB})$ \\
\hline 0,80 & 0,15 & 7,49 & 0,01 & ELV (PB) & 0,80 & 0,15 & 9,34 & 0,01 & ELV (PB) \\
\hline 0,90 & 0,075 & 15,25 & 0,02 & ELV (PO) & 0,90 & 0,075 & 16,92 & 0,01 & ELV (PO) \\
\hline 0,95 & 0,038 & 17,88 & 0,02 & $\mathrm{ELV}(\mathrm{PO})$ & 0,95 & 0,038 & 19,76 & 0,02 & $\mathrm{ELV}(\mathrm{PO})$ \\
\hline \multicolumn{10}{|c|}{ Fração molar AO:acetato etila de 1:9 } \\
\hline \multicolumn{5}{|c|}{$T=35^{\circ} \mathrm{C}$} & \multicolumn{5}{|c|}{$T=45^{\circ} \mathrm{C}$} \\
\hline
\end{tabular}




\begin{tabular}{|c|c|c|c|c|c|c|c|c|c|}
\hline 0,50 & 0,45 & 3,34 & 0,01 & ELV (PB) & 0,50 & 0,45 & 4,02 & 0,02 & ELV (PB) \\
\hline 0,60 & 0,36 & 4,45 & 0,03 & ELV (PB) & 0,60 & 0,36 & 4,52 & 0,03 & $\mathrm{ELV}(\mathrm{PB})$ \\
\hline 0,70 & 0,27 & 4,62 & 0,01 & ELV (PB) & 0,70 & 0,27 & 5,59 & 0,02 & ELV (PB) \\
\hline 0,80 & 0,18 & 5,55 & 0,03 & ELV (PB) & 0,80 & 0,18 & 6,81 & 0,01 & $\mathrm{ELV}(\mathrm{PB})$ \\
\hline 0,85 & 0,13 & 6,14 & 0,01 & ELV (PB) & 0,85 & 0,13 & 7,27 & 0,01 & $\mathrm{ELV}(\mathrm{PB})$ \\
\hline 0,90 & 0,090 & 6,48 & 0,02 & ELV (PB) & 0,90 & 0,090 & 8,83 & 0,02 & $\mathrm{ELV}(\mathrm{PB})$ \\
\hline 0,95 & 0,045 & 13,37 & 0,01 & ELV (PB) & 0,95 & 0,045 & 15,66 & 0,03 & $\mathrm{ELV}(\mathrm{PB})$ \\
\hline \multicolumn{5}{|c|}{$T=55^{\circ} \mathrm{C}$} & \multicolumn{5}{|c|}{$T=65^{\circ} \mathrm{C}$} \\
\hline 0,50 & 0,45 & 4,25 & 0,03 & ELV (PB) & 0,50 & 0,45 & 4,93 & 0,01 & $\operatorname{ELV}(\mathrm{PB})$ \\
\hline 0,60 & 0,36 & 5,31 & 0,03 & ELV (PB) & 0,60 & 0,36 & 6,28 & 0,04 & $\mathrm{ELV}(\mathrm{PB})$ \\
\hline 0,70 & 0,27 & 6,58 & 0,01 & ELV (PB) & 0,70 & 0,27 & 7,62 & 0,03 & ELV (PB) \\
\hline 0,80 & 0,18 & 7,87 & 0,02 & ELV (PB) & 0,80 & 0,18 & 9,19 & 0,02 & $\operatorname{ELV}(\mathrm{PB})$ \\
\hline 0,85 & 0,13 & 8,61 & 0,01 & ELV (PB) & 0,85 & 0,13 & 10,14 & 0,01 & ELV (PO) \\
\hline 0,90 & 0,090 & 11,33 & 0,01 & ELV (PO) & 0,90 & 0,090 & 13,75 & 0,01 & $\mathrm{ELV}(\mathrm{PO})$ \\
\hline 0,95 & 0,045 & 18,28 & 0,01 & ELV (PO) & 0,95 & 0,045 & 21,35 & 0,01 & ELV (PO) \\
\hline \multicolumn{10}{|c|}{ Fração molar AO:acetato etila de 1:200 } \\
\hline \multicolumn{5}{|c|}{$T=35^{\circ} \mathrm{C}$} & \multicolumn{5}{|c|}{$T=45^{\circ} \mathrm{C}$} \\
\hline 0,50 & 0,49 & 3,45 & 0,02 & ELV (PB) & 0,50 & 0,49 & 3,81 & 0,01 & $\mathrm{ELV}(\mathrm{PB})$ \\
\hline 0,60 & 0,40 & 4,03 & 0,03 & ELV (PB) & 0,60 & 0,40 & 4,87 & 0,03 & ELV (PB) \\
\hline 0,70 & 0,30 & 4,70 & 0,01 & ELV (PB) & 0,70 & 0,30 & 5,59 & 0,02 & $\operatorname{ELV}(\mathrm{PB})$ \\
\hline 0,80 & 0,20 & 5,88 & 0,02 & ELV (PB) & 0,80 & 0,20 & 6,58 & 0,02 & $\operatorname{ELV}(\mathrm{PB})$ \\
\hline 0,85 & 0,15 & 5,83 & 0,01 & ELV (PO) & 0,85 & 0,15 & 7,01 & 0,01 & ELV (PO) \\
\hline 0,90 & 0,1 & 6,56 & 0,01 & ELV (PO) & 0,90 & 0,1 & 7,62 & 0,01 & ELV (PO) \\
\hline 0,95 & 0,05 & 7,00 & 0,01 & ELV (PO) & 0,95 & 0,05 & 8,20 & 0,02 & ELV (PO) \\
\hline \multicolumn{5}{|c|}{$T=55^{\circ} \mathrm{C}$} & \multicolumn{5}{|c|}{$T=65^{\circ} \mathrm{C}$} \\
\hline 0,50 & 0,49 & 4,43 & 0,01 & ELV (PB) & 0,50 & 0,49 & 4,96 & 0,01 & $\mathrm{ELV}(\mathrm{PB})$ \\
\hline 0,60 & 0,40 & 5,34 & 0,01 & ELV (PB) & 0,60 & 0,40 & 6,12 & 0,02 & ELV (PB) \\
\hline 0,70 & 0,30 & 6,47 & 0,01 & ELV (PB) & 0,70 & 0,30 & 7,41 & 0,01 & ELV (PB) \\
\hline 0,80 & 0,20 & 7,53 & 0,02 & ELV (PB) & 0,80 & 0,20 & 8,67 & 0,02 & $\operatorname{ELV}(\mathrm{PB})$ \\
\hline 0,85 & 0,15 & 8,13 & 0,01 & ELV (PO) & 0,85 & 0,15 & 9,42 & 0,02 & ELV (PO) \\
\hline 0,90 & 0,1 & 9,03 & 0,03 & ELV (PO) & 0,90 & 0,1 & 10,15 & 0,01 & ELV (PO) \\
\hline 0,95 & 0,05 & 9,47 & 0,01 & ELV (PO) & 0,95 & 0,05 & 10,68 & 0,01 & ELV (PO) \\
\hline
\end{tabular}

Nas Figuras 1 e 2 é possível observar graficamente os resultados experimentais obtidos para o 
sistema estudado. Nas duas primeiras projeções (Figura 1 e 2) se observa que, ao aumentar a fração de $\mathrm{CO}_{2}$, ocorre um aumento na pressão da transição de fase. Em frações altas de $\mathrm{CO}_{2}($ acima de 0,9$)$ se observa um grande aumento nas pressões de transição de fase. Para cada uma das isotermas representadas, a região acima da curva dos pontos experimentais representa uma região com uma única fase. Abaixo da curva coexistem uma fase líquida e outra vapor. O sistema estudado, nas duas frações molares de acetato de etila e $\mathrm{AO}$ apresentam boa solubilidade em $\mathrm{CO}_{2}$ em pressões relativamente baixas.

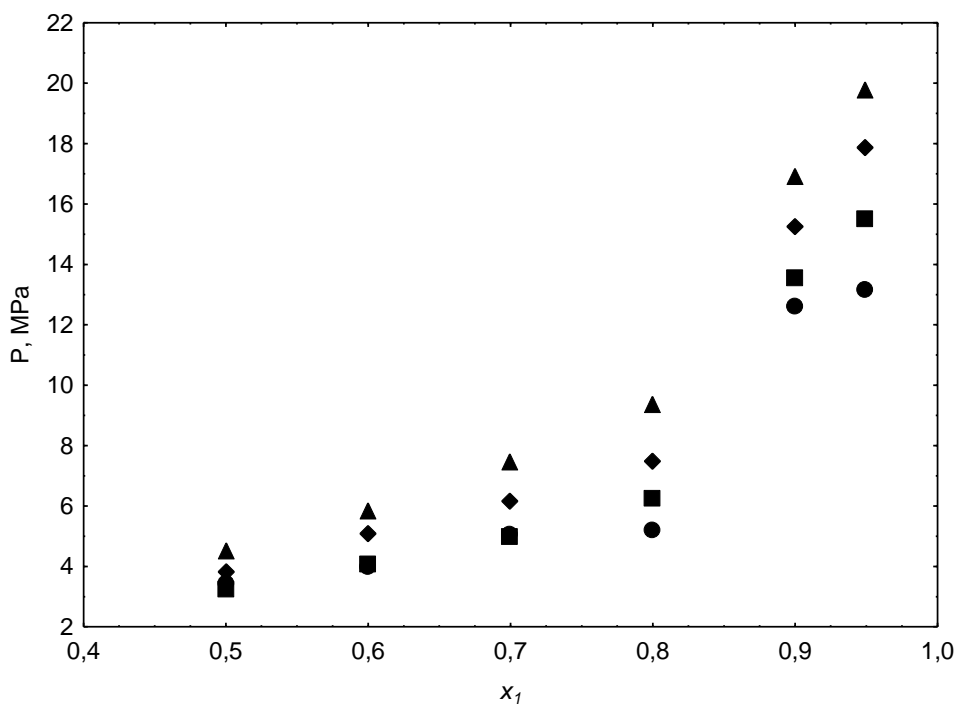

Figura 1 - Diagrama da pressão-composição do sistema $\left\{\mathrm{CO}_{2}(1)+(0,75\right.$ acetato de etila(2) $+0,25$ ácido oleico(3)\} a $\mathrm{T}=35^{\circ} \mathrm{C}(\bullet) ; 45^{\circ} \mathrm{C}(\boldsymbol{\square}) ; 55^{\circ} \mathrm{C}(\bullet) ; 65^{\circ} \mathrm{C}(\boldsymbol{\Delta})$.

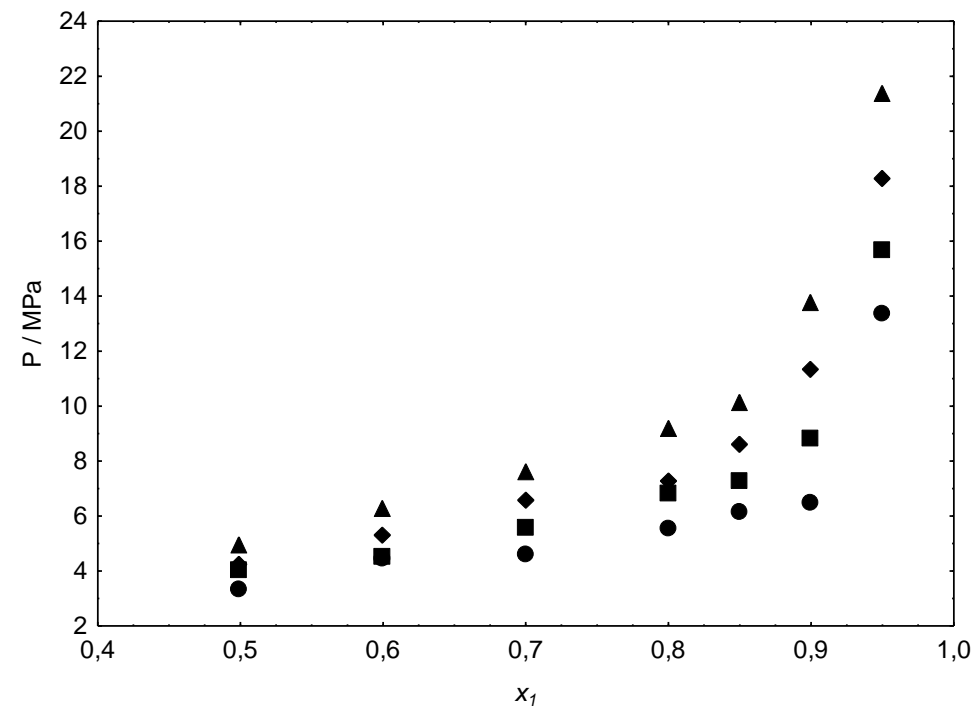

Figura 2 - Diagrama da pressão-composição do sistema $\left\{\mathrm{CO}_{2}(1)+(0,90\right.$ acetato de etila(2) + 0,10 ácido oleico(3)\} a $\mathrm{T}=35^{\circ} \mathrm{C}(\bullet) ; 45^{\circ} \mathrm{C}(\boldsymbol{\square}) ; 55^{\circ} \mathrm{C}(\bullet) ; 65^{\circ} \mathrm{C}(\boldsymbol{\Delta})$. 
A Figura 3 compara o sistema ternário obtido neste trabalho $\left\{\mathrm{CO}_{2}(1)+(0,995\right.$ acetato de etila $(2)+0,005$ ácido oleico(3) $\}$ com dados encontrados na literatura de um sistema binário $\left\{\mathrm{CO}_{2}(1)\right.$ + acetato de etila(2)\} (SIMA et al.,, 2012). Como no sistema ternário a quantidade de ácido oleico é muito pequena, tal sistema se comporta como binário $\left(\mathrm{CO}_{2}+\right.$ acetato de etila), ou seja, pequenas concentrações de ácido oleico tem pouco efeito sobre as transições de fase.

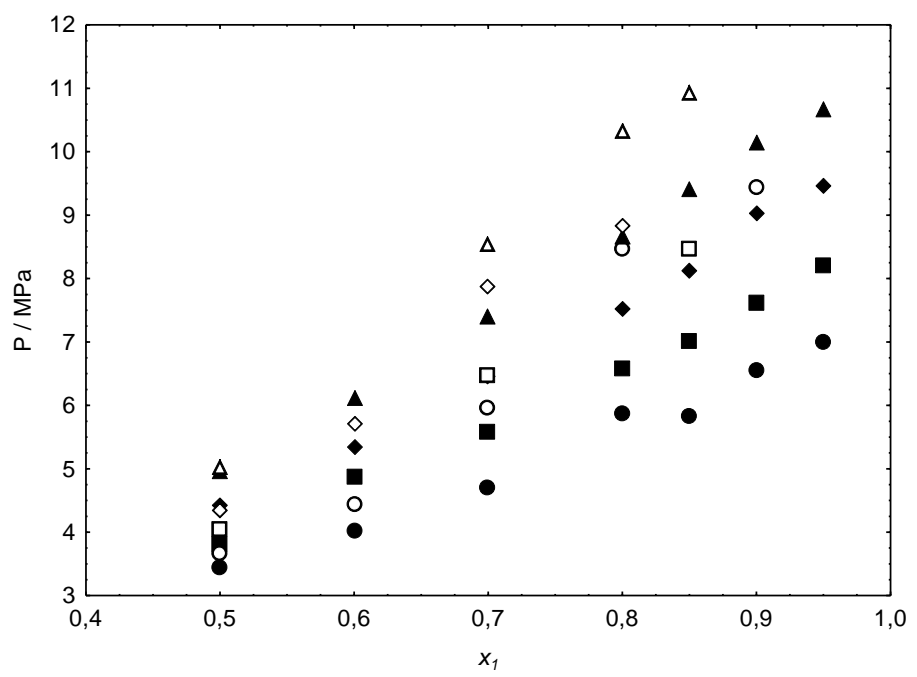

Figura 3 - Diagrama da pressão-composição: comparação com dados da literatura. Este trabalho, sistema ternário $\left\{\mathrm{CO}_{2}(1)+(0,995\right.$ acetato de etila(2) $+0,005$ ácido oleico(3) $\}: \bullet, 35^{\circ} \mathrm{C} ; \mathbf{\square}, 45^{\circ} \mathrm{C}$; $\bullet, 55^{\circ} \mathrm{C} ; \boldsymbol{\wedge}, 65^{\circ} \mathrm{C}$. SIMA et al., (2012), sistema binário $\left(\mathrm{CO}_{2}(1)+\right.$ acetato de etila $\left.(2)\right): \square, 50^{\circ} \mathrm{C} ; \square$, $60^{\circ} \mathrm{C} ; \square, 70^{\circ} \mathrm{C} ; \Delta, 80^{\circ} \mathrm{C}$.

\section{CONCLUSÃO}

Este trabalho relatou as medidas de transição de fase para o sistema $\left\{\mathrm{CO}_{2}(1)+\right.$ acetato de etila(2) + ácido oleico(3) \} a temperaturas de 35 a $65^{\circ} \mathrm{C}$. Nas composições estudadas, os componentes do sistema apresentam excelente solubilidade em $\mathrm{CO}_{2}$. Este fator é importante, pois permite concluir que a reação de formação de epóxidos a partir do acetato de etila e ácido oleico em $\mathrm{CO}_{2}$ supercrítico é favorecida. Os dados experimentais relatados neste trabalho são importantes informações para o desenvolvimento do novo processo de formação de epóxidos aqui sugerido.

\section{REFERÊNCIA BIBLIOGRÁFICA}

AKIN, O.; TEMELLI, F. Effect of supercritical $\mathrm{CO}_{2}$ pressure on polymer membranes. J.Memb. Sc., 399-400, 1-10, 2012.

AL-DARMAKI, N.; LUA, T.; AL-DURI, B.; HARRIS, J.B.; FAVRE, T.F.; BHAGGAN, K.; SANTOS, R.C.D. Solubility measurements and analysis of binary, ternary and quaternary systems of palm olein, squalene and oleic acid in supercritical carbon dioxide. Separ.Pur.Tech., 83, 189-195, 


\section{9 a 22 de outubro de 2014 \\ Florianópolis/SC}

2011.

CORREA, F. A.; SUTILI, F. K.; MIRANDA, L. S. M.; LEITE, S. G. F.; SOUZA, R. O. M. A.; LEAL, I. C. R. Epoxidation of oleic acid catalysed by PSCL-Amano lipase optimized by experimental design. J. Mol. Catal. B: Enzym., v. 81, p. 7-11, 2012.

DINDA, S.; GOUD, V. V.; PATWARDHAN, A. V.; PRADHAN, N. C. Selective epoxidation of natural triglycerides using acidic ion exchange resin as catalyst. Asia-Pacific J. Chem. Engin., v. 6, p. 870-878, 2011.

GIUFRIDA, W. M.; RODRIGUEZ-REARTES, S. B.; ALONSO, C. G.; ZABALOY, M. S.; CABRAL, V. F.; TAVARES, F. W.; CARDOZO-FILHO, L. High-Pressure Experimental Data of $\mathrm{CO} 2+$ Mitotane and $\mathrm{CO}_{2}+$ Ethanol + Mitotane Mixtures. J. Chem. Engin. Data, v. 56, p. 4333-4341, 2011.

KOTLEWSKA, A. J.; VAN RANTWIJK, F.; SHELDON, R. A.; ARENDS, I. W. C. E. Epoxidation and Baeyer-Villiger oxidation using hydrogen peroxide and a lipase dissolved in ionic liquids. Gr. Chemist., v. 13, p. 2154-2160, 2011.

LAUDANI, C.G.; PRIMOŽIČ, M.; KNEZ, Z.; HABULIN, M. Phase Equilibrium Measurements and Data Correlation for the Ternary System Oleic Acid + 1-Octanol + Carbon Dioxide. Croat. Chem. Acta 82 (4), 761-769, 2009.

LU, H.; SUN, S.; BI, Y.; YANG, G.; MA, R.; YANG, H. Enzymatic epoxidation of soybean oil methyl esters in the presence of free fatty acids. Eur. J.Lip. Sc. Technol., v. 112, p. 1101-1105, 2010.

MAZZER, H. R.; SANTOS, J. C. O.; CABRAL, V. F.; DARIVA, C.; KUNITA, M. H.; RUBIRA, A. F.; AZNAR, M.; CARDOZO-FILHO, L. Phase Behavior at High Pressure of the Ternary System: CO2, Ionic Liquid and Disperse Dye. J. Thermod., v. 2012, p. 1-6, 2012.

MUSHTAQ, M.; TAN, I.M.; NADEEM, M.; DEVI, C.; LEE, S.Y. C.; SAGIR, M.; RASHID, U. Epoxidation of methyl esters derived from Jatropha oil: An optimization study. Grasas y Aceites, v. 64(1), p. 103-114, 2013.

NOLEN, S. A.; LU, J.; BROWN, J. S.; POLLET, P.; EASON, B. C.; GRIFFITH, K. N.; GLASER, R.; BUSH, D.; LAMB, D. R.; LIOTTA, C. L.; ECKERT, C. A.; THIELE, G. F.; ECKERT, C. A. Olefin Epoxidations Using Supercritical Carbon Dioxide and Hydrogen Peroxide without Added Metallic Catalysts or Peroxy. Ind. Engin. Chemis. Res., v. 41, p. 316-323, 2002.

PETROVIC, Z. S.; ZLATANIC, A.; LAVA, C. C.; SINADINOVIC-FISER, S. Epoxidation of soybean oil in toluene with peroxoacetic and peroxoformic acids-kinetics and side reactions. Eur. J. Lip. Sc. Technol., v. 104, p. 293-299, 2002.

PINTO, L. F.; RODRIGUEZ-REARTES, S. B.; CORAZZA, M. L.; CABRAL, V. F.; ARAÚJO, P. H. H., MADUREIRA, E. H.; ZABALOY, M. S.; CARDOZO-FILHO, L. Phase behavior of carbon dioxide+medroxyprogesterone acetate system at high pressures. $\mathrm{Fl}$. $\mathrm{Ph}$. Equil., v. 349, p. 1-11, 2013.

RIOS, L. A.; WECKES, P.; SCHUSTER, H.; HOELDERICH, W. F. Mesoporous and amorphous Ti-silicas on the epoxidation of vegetable oils. J. Catal., v. 232, p. 19-26, 2005.

ROUMANET P-R.; LAFLÈCHE, F.; JARROUX, N.; RAOUL, Y.; CLAUDE, S.; GUÉGAN, P.Novel aliphatic polyesters from an oleic acid based monomer. Synthesis, epoxidation, cross-linking and biodegradation. European Polymer Journal, v. 49, p. 813-822, 2013

SAITHAI, P.; LECOMTE, J.; DUBREUCQ, E.; TANRATTANAKUL, V. Effects of different epoxidation methods of soybean oil on the characteristics of acrylated epoxidized 
soybean oil-co-poly(methyl methacrylate) copolymer. Express Polymer Letters, v.7(11), p. 910924, 2013.

SANTOS, J. C.; MAZZER, H. R.; MACHADO, G.D.; ANDREAUS, J.; CABRAL, V. F.; ZABALOY, M.S.; CARDOZO-FILHO, L. High-pressure phase behaviour of the system $\left(\mathrm{CO}_{2}+\right.$ C.I. Disperse Orange 30 dye). J.Chem. Thermod., v. 48, p. 284-289, 2012.

SILVA, W. S. D.; LAPISC, A. A. M.; SUAREZA, P. A. Z.; NETO, B. A. D. Enzymemediated epoxidation of methyl oleate supported by imidazolium-based ionic liquids. J. Mol. Catal. B: Enzyma., v. 68, p. 98-103, 2011.

SIMA, S.; FEROIU, V.; GEAN, D. New high pressure vapor-liquid equilibrium data and density predictions for carbon dioxide + ethyl acetate system. Fl. Ph. Equil., v. 325, p. 45-52, 2012.

SUN, S.; KE, X.; CUI, L.; YANG, G.; BI, Y.; SONG, F.; XU, X. Enzymatic epoxidation of Sapindus mukorossi seed oil by perstearic acid optimized using response surface methodology. Ind. Crops Prod., v. 33, p. 676-682, 2011. 